\title{
Arterial stiffness in adults with steady-state bronchiectasis: association with clinical indices and disease severity
}

\author{
Yong-hua Gao ${ }^{1 \dagger}$, Juan-juan Cuil ${ }^{1 \dagger}$, Ling-yun Wang ${ }^{2}$, Ke-qin Yin', Li Wang ${ }^{1}$, Guo-jun Zhang ${ }^{1 \dagger}$ and Shao-xia Liu ${ }^{1 *}$
}

\begin{abstract}
Background: Cardiovascular disease are common co-morbidities in bronchiectasis and contribute substantially to disease burden and mortality. Brachial-ankle pulse wave velocity (baPWV), a measure of arterial stiffness, has a strong predictive value for cardiovascular event. We hypothesized that baPWV would be increased in steady-state bronchiectasis patients, and correlates with the degree of systemic inflammation and disease severity assessed with Bronchiectasis Severity Index and FACED scores.

Methods: Eighty patients with steady-state bronchiectasis and 80 age- and sex-matched controls were enrolled. BaPWV was measured as an indicator of arterial stiffness. Demographic, clinical indices, radiology, spirometry, sputum bacteriology and systemic inflammatory mediators were also assessed.

Results: Bronchiectasis patients had significantly increased baPWV [median $1514 \mathrm{~cm} / \mathrm{s}$ vs. $1352 \mathrm{~cm} / \mathrm{s}, P=0.0003$ ] compared with control subjects. BaPWV significantly correlated with Bronchiectasis Severity Index (rho $=0.65, P<0.001$ ) and FACED (rho $=0.49, P<0.001)$ scores. In multivariate regression analysis, age, Pseudomonas aeruginosa colonization, systolic blood pressure, body-mass index and exacerbation frequency in the last 12 months, but not systemic inflammatory markers, were independent factors influencing on baPWV in bronchiectasis patient after adjustment for other clinical variables. Reproducibility of baPWW measurement was good.

Conclusion: Bronchiectasis patients have increased arterial stiffness compared with control subjects, which correlates with disease severity, but not systemic inflammatory markers. Age, Pseudomonas aeruginosa colonization, systolic blood pressure, body-mass index and exacerbation frequency in last 12 months might independently predict the severity of arterial stiffness in bronchiectasis. Therefore, arterial stiffness might have contributed to the increased risks of developing cardiovascular diseases in bronchiectasis.
\end{abstract}

Keywords: Bronchiectasis, Arterial stiffness, Pulse wave velocity, Systemic inflammation, Cardiovascular disease

\section{Background}

Bronchiectasis is a chronic airway disease characterized by recurrent infection and chronic inflammation. The prevalence of bronchiectasis has been increasing over the past decade and led to substantial morbidity and mortality worldwide [1-3]. Recent studies have demonstrated that patients with bronchiectasis have increased risks of developing cardiovascular disease (CVD), and

\footnotetext{
* Correspondence: 375891371@qq.com

${ }^{\dagger}$ Equal contributors

'Department of Respiratory and Critical Care Medicine, The First Affiliated Hospital of Zhengzhou University, 1 Jianshe East Road, Zhengzhou 450052, Henan, China

Full list of author information is available at the end of the article
}

that concomitant CVD might pose a negative impact on survival [4-7]. The underlying mechanisms of elevated cardiovascular risk are still unclear, but increased systemic inflammation and frequent exacerbations due to chest infections might play a crucial role $[5,8]$.

Arterial stiffness reflects the decreased capability of an artery to dilate and contract in response to pressure changes. A multitude of non-invasive methods have been developed to assess arterial stiffness $[9,10]$. Of these, pulse-wave velocity (PWV) is the most widely used and validated technique [10]. The carotid-femoral PWV (cfPWV) is the gold standard for assessing arterial stiffness $[9,11]$, but requires patients' persistent lateral 
rotation of the neck and exposure of inguinal region [12]. However, brachial-ankle PWV (baPWV) has been extensively applied for assessment in East Asian countries (including China), presumably due to the greater ease and convenience over a longer arterial length, and recent evidence has shown similar usefulness of baPWV in CVD risk stratification compared with cfPWV [13].

Previous studies suggested that individuals with chronic respiratory diseases have increased arterial stiffness compared with age- and sex-matched control subjects [14-16]. This has led us to postulate that similar conclusions apply to bronchiectasis patients because they share some common biological pathways for developing CVD (i. e. hypoxia and systemic inflammation) $[8,17]$. In a small, single-center study (20 patients versus 20 controls), Gale and colleagues [18] have shown that bronchiectasis patients had greater arterial stiffness, but they defined arterial stiffness by measuring aortic PWV. Bronchiectasis Severity Index (BSI) and FACED score have been widely used to categorize the severity of bronchiectasis $[19,20]$. However, their relationship with arterial stiffness remained unclear. In addition, bronchiectasis exacerbations are common and important events related to exaggerated airway infection and systemic inflammation [21, 22], which might accelerate the process of impairment of the cardiovascular system.

Therefore, we aimed to: (1) assess arterial stiffness in bronchiectasis patients by measuring baPWV as compared with age- and sex-matched healthy controls; (2) elucidate the relationship between disease severity and arterial stiffness; and (3) determine the factors influencing on arterial stiffness in patients with bronchiectasis.

\section{Methods}

\section{Study population}

Eighty-nine steady-state bronchiectasis patients (defined as no major deterioration in clinical signs and symptoms within 4 weeks) aged 18 years or greater were prospectively recruited from the pulmonology clinic of The First Hospital of Zhengzhou University between January 2016 and March 2017. Patients were enrolled if they had a clinical diagnosis of bronchiectasis based on high-resolution computed tomography (HRCT) combined with a compatible history (i.e. chronic cough, sputum production or hemoptysis etc.). Subjects were excluded if they (1) had severe cardiovascular disease (i.e. uncontrolled congestive heart failure, unstable angina pectoris or myocardial infarction within 3 months); (2) had uncontrolled hypertension [systolic blood pressure (SBP) $\geq 160 \mathrm{mmHg}$ and/or diastolic blood pressure (DBP) $\geq 90 \mathrm{mmHg}$; (3) had renal or hepatic dysfunction; (4) had malignancy; (5) refused to perform baPWV measurement. Patients with traction bronchiectasis due to severe emphysema or advanced fibrosis, and uncontrolled asthma were excluded. Finally, a total of 80 subjects were included for the analysis. Meanwhile, 80 healthy subjects with normal chest X-ray and no prior history or symptoms of chronic respiratory diseases (including asthma, chronic obstructive pulmonary disease, etc.) who were individually matched with bronchiectasis patients for age and sex were recruited during the same period from the Health Check-up Center. Ethical approval was obtained by the Ethics Committee of The First Affiliated Hospital of Zhengzhou University. Written informed consent was obtained.

\section{History inquiry and disease severity assessment}

Data collection included demographic data, anthropometric measures, smoking history, comorbidities, the number of exacerbations and hospitalizations in the previous year, current treatments. Exacerbation was defined as a significant increase in respiratory symptoms (increased cough frequency, increased sputum volume or viscosity, increased sputum purulence with or without increased wheezing, breathlessness, haemoptysis) and/or systemic upset requiring antibiotic treatment [23]. Exacerbation history was recorded according to patient recall, followed by verification of available medical charts. Chest HRCT scan within 12 months was used for radiologic scoring based on the number of bronchiectatic lobes (with the lingula being scored as a separate lobe) and the severity of bronchial dilatation (tubular: 1 point, varicose: 2 points, cystic: 3 points), with the maximal score of 18 [20]. Aetiology of bronchiectasis was determined as previously described [24, 25]. Cystic fibrosis and $a 1$ anti-trypsin deficiency have been scarcely reported in Asian countries, therefore routine screening was not conducted [24].

Disease severity was assessed with both BSI and FACED score $[19,20]$, respectively. The items of BSI included age, body-mass index (BMI), exacerbation frequency, prior hospitalization, MRC dyspnea score, forced expiratory volume in one second $\left(\mathrm{FEV}_{1}\right)$ predicted\%, Pseudomonas aeruginosa (PA) colonization, colonization with other potentially pathogenic microorganisms (PPMs) and the number of bronchiectatic lobes, The BSI of $0-4,5-8$, and 9 or greater corresponded to mild, moderate and severe bronchiectasis, respectively. FACED score consisted of $\mathrm{FEV}_{1}$ predicted\%, age, PA colonization, radiological extension and MRC dyspnea score. The FACED score of $0-2,3-4$, and 5-7 corresponded to mild, moderate and severe bronchiectasis, respectively.

\section{Laboratory assessment}

Venous blood was drawn from bronchiectasis patients after fasting overnight for at least $8 \mathrm{~h}$ before venipuncture. Total cholesterol, triglycerides and C-reactive protein were measured by standardized and certified program using automatic biochemical analyzer (AU5800, BECKMAN 
COULTER, USA). Serum interleukin (IL)-6 and IL-8 were measured using commercial multiplex bead-based assay kits described previously (Bio-Plex Cytokines Assay; Bio-Rad Inc) [23]. Citrated plasma samples were analyzed for fibrinogen using the Clauss method (IL ACL Top Coagulation Analyzer; Instrumentation Laboratories, Lexington, MA).

\section{Sputum bacteriology and spirometry}

Fresh sputum was sent for culture within $2 \mathrm{~h}$ after removing oral cavity debris. Hypertonic saline (3\% 5\%) induction was applied when appropriate. Colonization of PA and other PPMs was defined as sputum culture positive for two or more occasions (at 3-month interval) for the same pathogenic bacteria within one year [23].

At baseline, all steady-state bronchiectasis patients underwent spirometry (QUARK PFT, COSMED Inc., Italy) according to international guidelines [26]. Results were originated from three technically repeatable maneuvers, with between-maneuver variation $<5 \%$ or $200 \mathrm{ml}$ in forced vital capacity (FVC) and FEV. The best $\mathrm{FVC}$ and $\mathrm{FEV}_{1}$ were reported.

\section{Measurement of baPWV}

A well-trained examiner (J.J.C.) measured baPWV among bronchiectasis patients using a non-invasive vascular screening device (BP-203RPE III, OMRON, Japan) according to manufacturer's instructions. Participants have rested for at least $10 \mathrm{~min}$ in supine position before measurement. A plethysmographic sensor was connected to the cuffs, which was wrapped around the brachia and ankles, to record simultaneously pulse volume waveforms. The time interval $(\Delta T)$ from the wave fronts of the brachial to ankle waveforms was recorded. The length between brachium and ankle $(\Delta \mathrm{L})$ was adjusted for participant's height [9]. The baPWV was calculated for each side by using the formula: baPWV $=\Delta \mathrm{L} / \Delta \mathrm{T}$ $(\mathrm{cm} / \mathrm{s})$. The average of baPWV for both sides was used for subsequent analyses. Higher baPWV corresponded to greater arterial stiffness and cardiovascular risk [9].

Reproducibility was assessed by measurement of baPWV between baseline and 2 weeks apart (from 8 a. $\mathrm{m}$. to 12 a.m.). Of the initial 20 bronchiectasis patients invited to participate the reproducibility study, two patients dropped out and one was excluded due to exacerbation during 2-week follow-up. Therefore, 17 patients (12 women and 5 men) were included in reproducibility analysis, with a mean age of 47.6 years. Notably, treatment strategies did not change at 2-week interval. The majority of patients were categorized as having moderate-to-severe bronchiectasis as assessed with the BSI, and three patients were classified as having mild bronchiectasis. Notably, patients were required to avoid physical activity, tobacco smoke for at least $12 \mathrm{~h}$, and vitamin supplementation for $72 \mathrm{~h}$ prior to each baPWV measurement.

\section{Statistical analysis}

Data were presented as mean \pm standard deviation (SD), median (interquartile range, IQR) or frequencies (proportion) as appropriate. Categorical variables were compared using $x^{2}$ tests. For continuous variables, two-group comparisons were performed using unpaired t tests or MannWhitney tests when appropriate. Spearman's correlation coefficients and intraclass correlation coefficients were used to compare baPWV values at baseline versus 2-week interval. Bland-Altman plot was used to demonstrate the repeatability of baPWV over time. Univariate and multivariate linear regression analysis were used to examine factors affecting baPWV in bronchiectasis patients. Cutoffs of $P$ values $<0.1$ in univariate models were initially eligible in multivariate model and removed using backward selection. The correlation between baPWV and bronchiectasis severity was determined with Spearman's rank or Pearson's correlation coefficient when appropriate. $P<0.05$ was considered statistically significant. All statistical analyses were performed using SPSS 16.0 (Chicago, IL, USA) and Graphpad Prism, version 5.0 (GraphPad Software Inc).

\section{Results}

\section{Subject characteristics}

Eighty patients and 80 age- and sex-matched controls were included for analysis. Baseline characteristics are shown in Table 1. Most patients were female (72.5\%), with a mean age of 51 years. Consistent with previous reports, [24, 25] the main aetiology of bronchiectasis was idiopathic or post-infectious disease. Concomitant airway disease was common [14 (17.5\%) and 5 (6.3\%) had COPD and asthma, respectively]. Seventeen patients (21.3\%) had PA colonization at baseline. No patients needed long-term oxygen therapy. Age, sex distribution and comorbidities did not differ between the two groups (all $P>0.05$ ).

\section{Reproducibility of baPWV in bronchiectasis}

The mean \pm SD baPWV of 17 bronchiectasis patients who had baPWV measurement at baseline and 2-week interval were $1507.18 \pm 264.04 \mathrm{~cm} / \mathrm{s}$ and $1544.41 \pm 240.71 \mathrm{~cm} / \mathrm{s}$, respectively $(P=0.67)$. The Bland-Altman plot of baPWV is shown in Fig. 1; the difference of baPWV was - 37.24 (156.70) $\mathrm{cm} / \mathrm{s}$. The Pearson correlation coefficient was $0.81(P<0.001)$ and intra-class correlation coefficient for baPWV was $0.89(0.71-0.96)$, suggesting a good reproducibility.

\section{Arterial stiffness assessment}

Patients with bronchiectasis had significantly higher baPWV than control subjects [median (IQR); $1514(1362-1718) \mathrm{cm} / \mathrm{s}$ 
Table 1 Baseline characteristics of subjects with steady-state bronchiectasis and healthy controls

\begin{tabular}{|c|c|c|c|}
\hline & Bronchiectasis & Controls & $P$ values \\
\hline Age, y & $51.0 \pm 13.9$ & $49.5 \pm 11.9$ & 0.47 \\
\hline Male sex, No. (\%) & $22(27.5)$ & $22(27.5)$ & 1.00 \\
\hline Body-mass index, $\mathrm{kg} / \mathrm{m}^{2}$ & $20.5 \pm 2.0$ & $25.2 \pm 3.6$ & $<0.001$ \\
\hline Current smoking, No. (\%) & $12(15.0 \%)$ & $18(22.5 \%)$ & 0.22 \\
\hline SBP, mmHg & $120.9 \pm 9.6$ & $117.6 \pm 11.2$ & 0.62 \\
\hline $\mathrm{DBP}, \mathrm{mmHg}$ & $81.6 \pm 8.6$ & $73.4 \pm 10.5$ & 0.34 \\
\hline $\mathrm{HR}$, bpm & $82.6 \pm 5.3$ & $80.1 \pm 4.2$ & 0.001 \\
\hline \multicolumn{4}{|l|}{ Spirometry } \\
\hline FVC\% predicted & $82.3 \pm 16.7$ & - & - \\
\hline $\mathrm{FEV}_{1} \%$ predicted & $67.3 \pm 21.4$ & - & - \\
\hline mMRC grade & $1.0(0-2.0)$ & - & - \\
\hline $\begin{array}{l}\text { Exacerbation frequency in } \\
\text { the past } 12 \mathrm{mo}\end{array}$ & $2.0(1.3-5.0)$ & - & - \\
\hline $\begin{array}{l}\text { Hospital admission in the } \\
\text { past } 24 \text { mo }\end{array}$ & $2.0(1.0-3.0)$ & - & - \\
\hline $\begin{array}{l}\text { Modiffied Reiff score for } \\
\text { chest HRCT scan }\end{array}$ & $5.0(2.0-10.0)$ & - & - \\
\hline PA colonization, No. (\%) & $17(21.3 \%)$ & - & - \\
\hline \multicolumn{4}{|l|}{ Aetiology } \\
\hline Idiopathic, No. (\%) & $26(32.5 \%)$ & - & - \\
\hline Post infective, No. (\%) & $25(31.3 \%)$ & - & - \\
\hline COPD, No. (\%) & $14(17.5 \%)$ & - & - \\
\hline Asthma, No. (\%) & $5(6.3 \%)$ & - & - \\
\hline Others $^{\mathrm{a}}$, No. (\%) & $10(12.5 \%)$ & - & - \\
\hline \multicolumn{4}{|l|}{ BSI Scores } \\
\hline Mild (0-4), No. (\%) & $27(33.8 \%)$ & - & - \\
\hline Moderate (5-8), No. (\%) & $11(13.8 \%)$ & - & - \\
\hline Severe ( $\geq 9)$, No. $(\%)$ & $42(52.5 \%)$ & - & - \\
\hline \multicolumn{4}{|l|}{ Comorbidities } \\
\hline Hypertension, No. (\%) & $1(1.3 \%)$ & $2(2.5 \%)$ & 0.56 \\
\hline Diabetes, No. (\%) & $6(7.5 \%)$ & $3(3.8 \%)$ & 0.30 \\
\hline $\begin{array}{l}\text { Coronary heart disease, } \\
\text { No. (\%) }\end{array}$ & $3(3.8 \%)$ & $3(3.8 \%)$ & 1.00 \\
\hline Stroke, No. (\%) & $0(0 \%)$ & $0(0 \%)$ & $\ldots$ \\
\hline $\begin{array}{l}\text { Peripheral arterial disease, } \\
\text { No. (\%) }\end{array}$ & $0(0 \%)$ & $0(0 \%)$ & $\ldots$ \\
\hline \multicolumn{4}{|l|}{ Clinical chemistry } \\
\hline Cholesterol, mmol/L & $3.8 \pm 1.1$ & - & - \\
\hline Triglyceride, mmol/L & $1.0 \pm 0.7$ & - & - \\
\hline $\mathrm{LDL}-\mathrm{C}, \mathrm{mmol} / \mathrm{L}$ & $2.2(1.9-2.4)$ & - & - \\
\hline $\mathrm{HDL}-\mathrm{C}, \mathrm{mmol} / \mathrm{L}$ & $1.9(1.0-1.4)$ & - & - \\
\hline
\end{tabular}

Table 1 Baseline characteristics of subjects with steady-state bronchiectasis and healthy controls (Continued)

\begin{tabular}{|c|c|c|c|}
\hline & Bronchiectasis & Controls & $P$ value \\
\hline \multicolumn{4}{|l|}{ Systemic inflammatory markers } \\
\hline IL-6, pg/mL & $3.3(1.8-7.9)$ & - & - \\
\hline $\mathrm{IL}-8, \mathrm{pg} / \mathrm{mL}$ & $7.1(4.3-11.8)$ & - & - \\
\hline C-reactive protein, mg/dL & $1.17(0.3-3.9)$ & - & - \\
\hline Fibrinogen, g/L & $3.2 \pm 0.9$ & - & - \\
\hline
\end{tabular}

Data are depicted as mean $\pm S D$, median (interquartile range [IQR]) or $n(\%)$ $B S I$ bronchiectasis severity index, COPD chronic obstructive pulmonary disease, $D B P$ diastolic blood pressure, $F E V_{1}$ forced expiratory volume in one second, FVC forced vital capacity, HR heart rate, HRCT high-resolution computed tomography, IL interleukin, mo month, $m M R C$ modified Medical Research Council, PA Pseudomonas aeruginosa, SBP systolic blood pressure; $y=y e a r$ ${ }^{a}$ Aspiration $(n=2)$, Allergic Bronchopulmonary Aspergillosis $(n=3)$, Microscopic Polyangiitis $(n=1)$, Rheumatoid Arthritis $(n=1)$, Inflammatory Bowel Disease $(n=1)$, Kartagener's Syndrome $(n=1)$, Primary Immunodeficiency $(n=1)$

vs. $1352(1218-1535) \mathrm{cm} / \mathrm{s}, P<0.001$ ] (Fig. 2). Subgroup analysis by excluding subjects with hypertension, coronary heart disease or diabetes demonstrated similar findings, with bronchiectasis patients $(n=70)$ having higher baPWV $(P<0.001)$ than control subjects $(n=72)$ (Additional file 1: Figure S1). The level of baPWV when stratified by PA colonization is shown in Additional file 1: Figure S2. Patients with PA colonization had significantly higher baPWV compared with those without [median (IQR); $1718(1617-1864) \mathrm{cm} / \mathrm{s}$ vs. $1405(1328-1705) \mathrm{cm} / \mathrm{s}$, $P<0.001]$. Moreover, both right and left baPWV values were markedly higher in bronchiectasis patients than in control subjects (both $P<0.001$; Additional file 1: Table S1). To explore the impacts of disease severity on arterial stiffness, bronchiectasis severity was assessed with both BSI and FACED scores. We observed a significant correlation between baPWV and BSI score (rho $=0.65, P<0.001$; Fig. 3a). Similar association was also applied for baPWV and FACED score (rho $=0.49, P<0.001$; Fig. $3 \mathrm{~b}$ ). Notably,

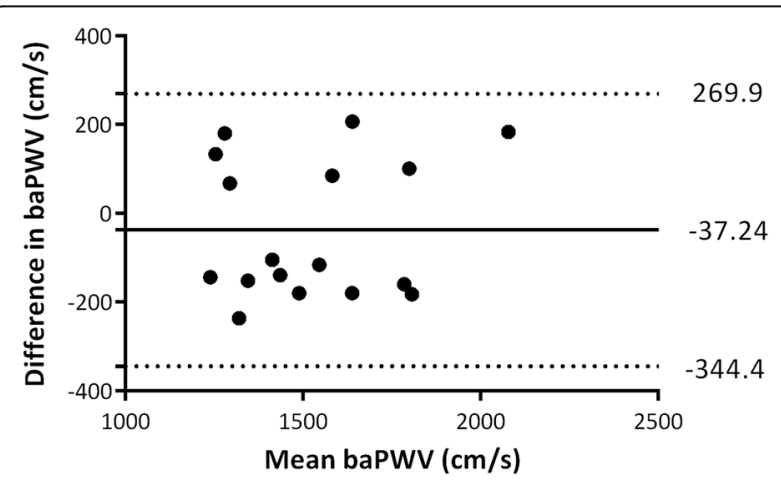

Fig. 1 Reproducibility of the baPWV. Bland-Altman plot of the baPWV over 2 weeks in 17 patients with steady-state bronchiectasis whose treatment remained unchanged. Solid line represents the mean difference of baPWV between the baseline and 2-week interval (- 37.24); Dotted line represents $95 \%$ limits of agreement $(-344.4$ to 269.9$)$ 


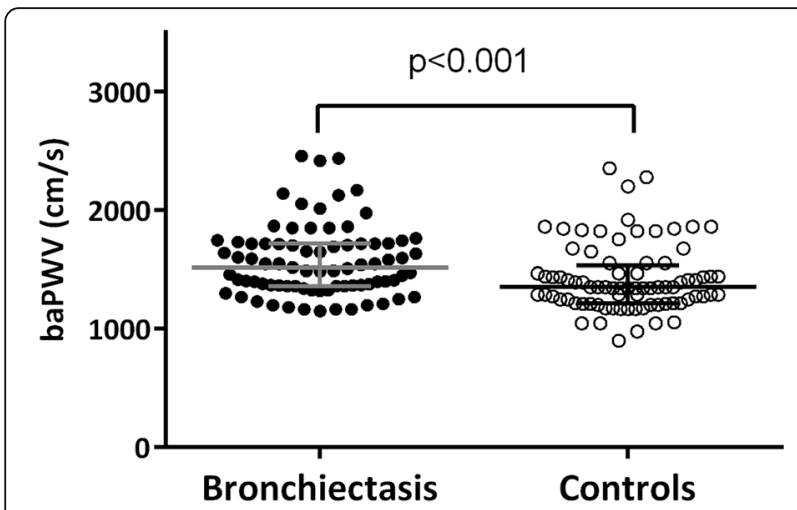

Fig. 2 The median (IQR) of baPW in patients with steady-state bronchiectasis $[1514(1362-1718) \mathrm{cm} / \mathrm{s}]$ and healthy controls [1352 $(1218-1535) \mathrm{cm} / \mathrm{s}]\left({ }^{*} P<0.001\right.$ by Mann-Whitney test). Significantly higher baPW was found in bronchiectasis patients compared with healthy controls

there was no significant correlation between systemic inflammatory markers [i.e. C-reactive protein (CRP), interleukin-6 (IL-6), IL-8 and fibrinogen] and the BSI (data not shown, all $P>0.05$ ).

\section{Determinants of baPWV in bronchiectasis}

In univariable analysis, factors that had a significant effect on baPWV included age, BMI, smoking history, $\mathrm{SBP}, \mathrm{FEV}_{1} \%$ predicted, $\mathrm{FVC} \%$ predicted, exacerbation frequency within 1 year, hospitalization frequency within 2 years, PA colonization, MRC dyspnea score, HRCT score and fibrinogen levels (Table 2). In multivariable analysis, age $(\beta=0.348, P<0.001)$, PA colonization $(\beta=0.282, P<0.001)$, SBP $(\beta=0.257, P=0.002)$, BMI $(\beta=0.250, P=0.001)$ and exacerbation frequency within one year $(\beta=0.195, P=0.07)$, but not systemic inflammatory markers, were independent factors influencing on the baPWV in bronchiectasis (Table 3).

\section{Discussion}

Our study found that bronchiectasis patients have increased baPWV compared with healthy controls, which was validated in subgroup analysis excluding individuals with concomitant hypertension or coronary heart disease or diabetes. BaPWV correlated with disease severity assessed with both BSI and FACED scores. Aging, PA colonization, SBP, BMI and exacerbation frequency in the previous year, but not systemic inflammatory markers, were independent factors affecting the baPWV in patient with bronchiectasis.

Recent studies have demonstrated that bronchiectasis might be an independent risk factor for CVD, which was unrelated to smoking-traditional cardiovascular risk factors or comorbidities associated with the etiology of bronchiectasis. This highlighted the importance of preventing from further development of CVD in
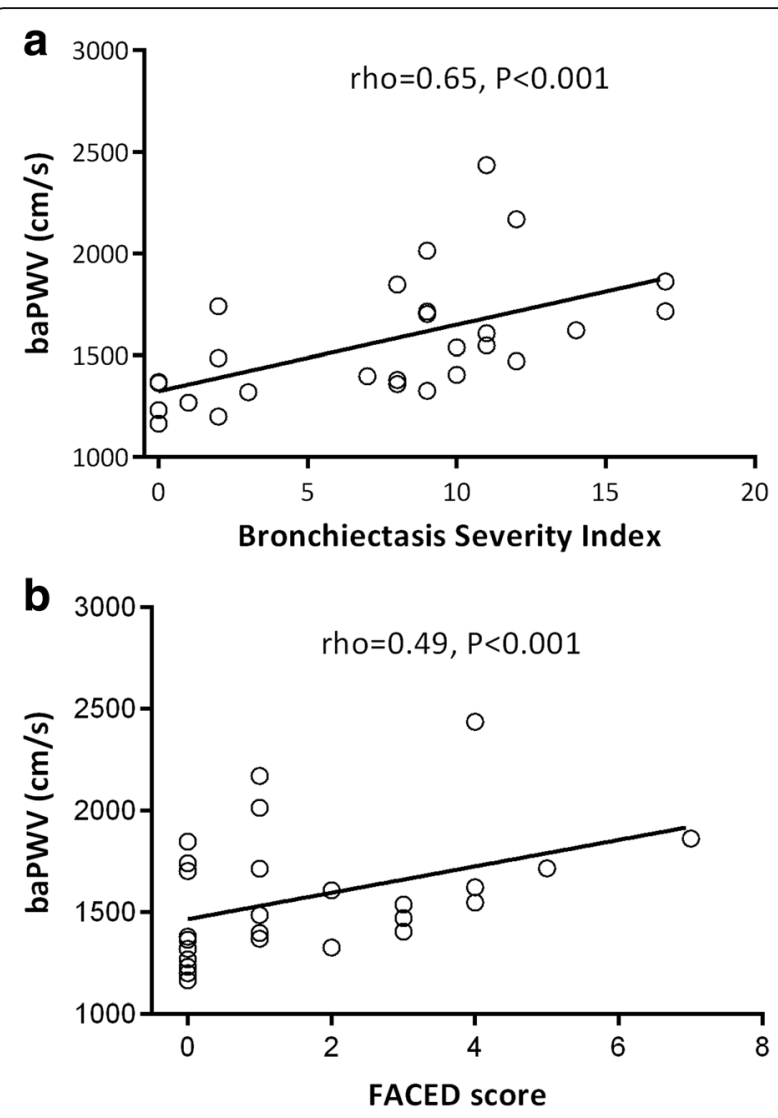

Fig. 3 The relationship between baPW and bronchiectasis severity assessed with BSI (rho $=0.65, P<0.001$ by Spearman's correlation analysis; (a) and FACED scores (rho $=0.49, P<0.001$ by Spearman's correlation analysis; (b). BaPW significantly correlated with BSI and FACED scores

bronchiectasis $[3-5,7]$. Thus, identifying the early changes of cardiovascular system before the occurrence of major clinical events (i.e. stroke and myocardial infarction), have additional implications for CVD prevention at individual levels, such as early-stage targeted prevention and intervention for high-risk patients. Arterial stiffness has a strong predictive value for cardiovascular events beyond traditional cardiovascular risk factors in healthy populations [9], as well as in patients with chronic lung diseases, and is the most suited parameter for routine clinical practice. Prior to our study, only one case-control study has reported that patient with bronchiectasis $(n=20)$ had increased arterial stiffness, measured by aortic PWV, compared with matched controls [18]. Similar results were also validated in our study despite using different indicators of arterial stiffness. We have, for the first time, demonstrated that baPWV correlated positively with the disease severity which was assessed with both the BSI and FACED scores, the twovalidated composite disease severity metrics, suggesting that arterial stiffness developed early in bronchiectasis 
Table 2 Results of univariable regression analysis for baPW of bronchiectasis patients

\begin{tabular}{|c|c|c|}
\hline Variable & $\beta$ & $P$ values \\
\hline \multicolumn{3}{|l|}{ Anthropometry } \\
\hline Age & 0.747 & $<0.001$ \\
\hline Male Sex & 0.214 & 0.056 \\
\hline $\mathrm{BMI}\left(\mathrm{kg} / \mathrm{m}^{2}\right)$ & 0.384 & $<0.001$ \\
\hline Smoking & 0.248 & 0.026 \\
\hline $\mathrm{SBP}, \mathrm{mmHg}$ & 0.617 & $<0.001$ \\
\hline $\mathrm{DBP}, \mathrm{mmHg}$ & 0.177 & 0.116 \\
\hline $\mathrm{HR}, \mathrm{bpm}$ & 0.108 & 0.341 \\
\hline \multicolumn{3}{|l|}{ Lung Function } \\
\hline $\mathrm{FEV}_{1} \%$ predicted & -0.468 & $<0.001$ \\
\hline FVC\% predicted & -0.478 & $<0.001$ \\
\hline \multicolumn{3}{|l|}{ Disease-related parameters } \\
\hline No. of exacerbations within 12 mo & 0.289 & 0.009 \\
\hline No. of hospitalization within 24 mo & 0.499 & $<0.001$ \\
\hline PA colonization & 0.442 & $<0.001$ \\
\hline Other bacterial colonization & -0.103 & 0.365 \\
\hline MRC dyspnea score & 0.249 & 0.026 \\
\hline HRCT score & 0.405 & $<0.001$ \\
\hline \multicolumn{3}{|l|}{ Comorbidities } \\
\hline COPD & 0.189 & 0.093 \\
\hline Coronary Heart Disease & 0.042 & 0.709 \\
\hline Diabetes & 0.217 & 0.054 \\
\hline \multicolumn{3}{|l|}{ Laboratory-related parameters } \\
\hline Cholesterol, mmol/L & 0.115 & 0.310 \\
\hline Triglyceride, mmol/L & -0.002 & 0.984 \\
\hline $\mathrm{LDL}-\mathrm{C}, \mathrm{mmol} / \mathrm{L}$ & 0.105 & 0.355 \\
\hline $\mathrm{HDL}-\mathrm{C}, \mathrm{mmol} / \mathrm{L}$ & -0.113 & 0.316 \\
\hline $\mathrm{IL}-6, \mathrm{pg} / \mathrm{mL}$ & 0.120 & 0.300 \\
\hline IL-8, pg/mL & 0.150 & 0.170 \\
\hline C-reactive protein, $\mathrm{mg} / \mathrm{dL}$ & 0.170 & 0.130 \\
\hline Fibrinogen, g/L & 0.249 & 0.026 \\
\hline
\end{tabular}

BaPWV brachial ankle pulse-wave velocity, $B M I$ body-mass index, COPD chronic obstructive pulmonary disease, DBP diastolic blood pressure, $F E V$, forced expiratory volume in one second, $F V C$ forced vital capacity, $H R$ heart rate, $H R C T$ high-resolution computed tomography, IL interleukin, $m M R C$ modified Medical Research Council, mo month, No number, SBP systolic blood pressure, $P A$ Pseudomonas aeruginosa, $y$ year

and aggravates along with greater disease severity or disease progression. A meta-analysis has shown that an increase in baPWV by $1 \mathrm{~m} / \mathrm{s}$ corresponded to an increase of 12 and $13 \%$ in the total cardiovascular events and cardiovascular mortality, respectively [27]. The elevation in baPWV between bronchiectasis patients and control subjects in our cohort was $1.62 \mathrm{~m} / \mathrm{s}$, which might result in an estimated increase of 19 and $21 \%$ in total cardiovascular events and cardiovascular mortality, respectively. In this
Table 3 Results of multiple regression analysis for baPWV of bronchiectasis patients

\begin{tabular}{lll}
\hline Variable & $\beta$ & $P$ \\
\hline Age & 0.348 & $<0.001$ \\
PA colonization & 0.282 & $<0.001$ \\
$\mathrm{SBP}, \mathrm{mmHg}$ & 0.257 & 0.002 \\
$\mathrm{BMI}\left(\mathrm{kg} / \mathrm{m}^{2}\right)$ & 0.250 & 0.001 \\
No. of exacerbations within 1 year & 0.195 & 0.007 \\
\hline BaPWV brachial ankle pulse-wave velocity, BMI body-mass index, PA Pseudomonas \\
aeruginosa, SBP systolic blood pressure
\end{tabular}

regard, this difference should be sufficient to elicit adverse outcomes in patients with bronchiectasis.

Global assessments of bronchiectasis severity with both BSI and FACED scores showed positive correlation with baPWV. This was further confirmed in our multivariate analysis that aging, PA colonization and the number of exacerbation within one year (all crucial components of the BSI) were independent factors influencing on baPWV in bronchiectasis. Recently, Evans et al. [5] have reported that bronchiectasis severity was independently associated with vascular disease, which was also consistent with our findings. Interestingly, the average age of bronchiectasis patients in our cohort was 51 years, which was significantly lower than that in European and US cohorts (70 years and 64 years, respectively) $[28,29]$. Since the fact that age was the strongest predictive factor of baPWV in our cohort, age-related changes in baPWV would be more prominent in European and US bronchiectasis cohorts, which merits further validations. Meanwhile, we found that PA colonization and the number of exacerbation within one year were significantly associated with arterial stiffness. The median difference of baPWV in patients with PA colonization compared to those without was $3.13 \mathrm{~m} / \mathrm{s}$ (unadjusted analysis), which corresponded to a $38 \%$ increase in excess risks of cardiovascular events [27]. Arterial stiffness was reportedly increased in cystic fibrosis children colonized with PA compared with uninfected children [30], which was consistent with our findings. PA colonization may represent a distinct clinical phenotype of bronchiectasis with poorer quality-of-life, frequent exacerbation and poorer long-term outcomes [31-33]. Our findings have extended the rationale to optimize management of patients with PA colonization, by integrating the concept of reduction in cardiovascular events. The association between exacerbation frequency and cardiovascular risk has been reported in COPD, and that chronic low-grade systemic inflammation might be one of the possible mechanisms linking frequent exacerbations to increased arterial stiffness [34]. However, the lack of association between systemic inflammatory markers and arterial stiffness in our study indicated that this assumption 
cannot be extrapolated to bronchiectasis patients. Nevertheless, further studies are merited to investigate the links between chronic bacterial infection, exacerbation, systemic inflammation and increased arterial stiffness by inclusion of greater sample sizes and longer follow-up duration.

Systemic inflammation has been proposed to accelerate and stimulate vascular extracellular matrix remodeling process of elastin fragmentation and collagen deposition, resulting in increased arterial stiffness [35]. However, data for the causal link between low-grade systemic inflammation and a higher incidence of cardiovascular disease in bronchiectasis are lacking [5]. In this study, although we found an association between baPWV and plasma fibrinogen, but not IL-6, IL-8 and CRP in univariate regression models, the results could not be further confirmed in multivariate model. Furthermore, systemic inflammatory markers did not correlate with bronchiectasis severity as assessed with BSI. However, we could not preclude the possibility that other known or unidentified inflammatory markers (i.e. intercellular adhesion molecule-1, vascular cell adhesion molecule, and E-selectin) might be related to arterial stiffness. Saleh et al. [36] recently shows that bronchiectasis patients presented with significantly heterogeneous levels of systemic inflammatory proteins, which could not be adequately accounted for by the differences in disease aetiology or severity. This indicated that persistent systemic inflammation may affect only some bronchiectasis patients as evidenced in COPD [37]. To further answer this question, it would be helpful to investigate exclusively in this specific subgroup of patients whether the correlation between inflammatory markers and increased arterial stiffness exists.

In addition, changes of arterial stiffness in bronchiectasis patients could not be explained by traditional cardiovascular risk factors, such as plasma lipids [38], diabetes [39], smoking history [40] and $\mathrm{FEV}_{1}$ [41], all powerful risk factors for predicting arterial stiffening. This might be because of a lack of effect of these risk factors per se, or because arterial stiffness was not driven by an atherosclerotic process at least in its early stages but rather by an alternative pathologic factor in which blood pressure plays a role [42]. It is well recognized that arterial stiffness depends on mechanical stretch of the arterial wall and, hence, on blood pressure at the time of the measurement [43]. Stretch is thought to transfer loading to stiffer elements within the wall that are of greater tensile strength (i.e. from elastin to collagen) and, therefore results in an overall stiffening of the wall. Accordingly, systolic blood pressure was independently associated with greater arterial stiffness in our study.

A major strength is that we have conducted the reproducibility of baPWV measurement and, for the first time, systematically investigated arterial stiffness in bronchiectasis patients. However, several limitations merit interpretation.
First, the study design was cross-sectional, and clinical follow-up data were not available, which precluded the causality inference between bronchiectasis, bacterial colonization, systemic inflammation, arterial stiffness and $\mathrm{CVD}$, and the direct comparison of predictive value of baPWV and cardiovascular risk scores for future cardiovascular events, which merits further investigation. Second, the inclusion of patients and controls subjects with $\mathrm{CHD}$, diabetes and hypertension might be criticized, although subgroup analysis with exclusion of these patients did not alter the findings. Thirdly, there are still some debates regarding whether COPD and asthma should be viewed as aetiologies of bronchiectasis [44], and abundant evidence of elevated arterial stiffness has been reported in COPD [14, 15]. We therefore conducted an exploratory subgroup analysis by removing patients with COPD or asthma in order to determine whether inclusion of these patients might have biased our findings. Reassuringly, baPWV remained significantly greater in bronchiectasis patients than those healthy controls (median; $1488 \mathrm{~cm} / \mathrm{s}$ versus $1352 \mathrm{~cm} / \mathrm{s}, P=0.004)$. Finally, left ventricular morphology and function were not assessed, which have been reportedly associated with arterial stiffness [45].

\section{Conclusions}

Patients with bronchiectasis had significantly higher baPWV compared with control subjects, mainly determined by age, PA colonization, SBP, BMI and the number of exacerbation in the last year. Disease severity, but not systemic inflammatory markers, was associated with the degree of baPWV. Further studies are needed to determine the usefulness of PWV measurement in risk stratification and clinical management of bronchiectasis patient to optimize cardiovascular outcomes.

\section{Additional file}

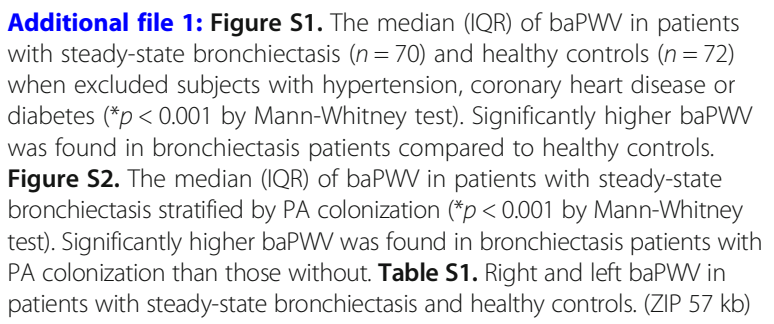

Additional file 1: Figure S1. The median ( $I Q R)$ of baPWV in patients with steady-state bronchiectasis $(n=70)$ and healthy controls $(n=72)$ when excluded subjects with hypertension, coronary heart disease or diabetes ( ${ }^{*} p<0.001$ by Mann-Whitney test). Significantly higher baPW was found in bronchiectasis patients compared to healthy controls. Figure S2. The median (IQR) of baPWW in patients with steady-state bronchiectasis stratified by PA colonization $\left({ }^{*} p<0.001\right.$ by Mann-Whitney test). Significantly higher baPW was found in bronchiectasis patients with PA colonization than those without. Table S1. Right and left baPW in patients with steady-state bronchiectasis and healthy controls. (ZIP $57 \mathrm{~kb}$ )

\section{Abbreviations}

BaPW: Brachial-ankle pulse wave velocity; BMI: Body-mass index; BSI: Bronchiectasis Severity Index; cfPWV: Carotid-femoral pulse-wave velocity; COPD: Chronic obstructive pulmonary disease; CVD: Cardiovascular disease;

DBP: Diastolic blood pressure; FEV1: Forced expiratory volume in one second; FVC: Forced vital capacity; HRCT: High-resolution computed tomography; IL: Interleukin; IQR: Interquartile range; PA: Pseudomonas aeruginosa; PPMs: Potentially pathogenic microorganisms; PWV: Pulse-wave velocity; SBP: Systolic blood pressure; SD: Standard deviation 


\section{Acknowledgements}

We wholeheartedly thank Wei-jie Guan (State Key Laboratory of Respiratory Disease, Guangzhou Institute of Respiratory Health, China) for his insights to improve the manuscript.

\section{Funding}

Dr. Gao declared that he has received the National Natural Science Foundation (81500006), and Open Project of State Key Laboratory of Respiratory Disease (SKLRD2016OP013)

\section{Availability of data and materials}

The data sets used and/or analyzed during the current study are available from the corresponding author on reasonable request.

\section{Authors' contributions}

Conceived and designed the experiments: YHG ZGJ and SXL; Performed the experiments: JJC, LYW, KQY, LW. Contributed reagents/materials/analysis tools: JJC, LYW, KQY, LW. Wrote the paper: YHG SXL. All authors read and approved the final manuscript.

\section{Ethics approval and consent to participate}

This study was approved by the institutional review boards of the First Affiliated Hospital of Zhengzhou University and all participants provided written informed consent.

\section{Competing interests}

The authors have reported to Respir Res that no potential conflicts of interest exist with any companies/organizations whose products or services may be discussed in this article.

\section{Publisher's Note}

Springer Nature remains neutral with regard to jurisdictional claims in published maps and institutional affiliations.

\section{Author details}

'Department of Respiratory and Critical Care Medicine, The First Affiliated Hospital of Zhengzhou University, 1 Jianshe East Road, Zhengzhou 450052. Henan, China. ${ }^{2}$ Department of Ultrasound Medicine, The First Affiliated Hospital of Zhengzhou University, Zhengzhou, Henan, China.

\section{Received: 5 February 2018 Accepted: 26 April 2018}

\section{Published online: 09 May 2018}

\section{References}

1. Ringshausen FC, de Roux A, Diel R, Hohmann D, Welte T, Rademacher J Bronchiectasis in Germany: a population-based estimation of disease prevalence. Eur Respir J. 2015;46:1805-7.

2. Quint JK, Millett ER, Joshi M, Navaratnam V, Thomas SL, Hurst JR, Smeeth L, Brown JS. Changes in the incidence, prevalence and mortality of bronchiectasis in the UK from 2004 to 2013: a population-based cohort study. Eur Respir J. 2016;47:186-93.

3. Roberts HJ, Hubbard R. Trends in bronchiectasis mortality in England and Wales. Respir Med. 2010;104:981-5.

4. Navaratnam V, Millett ER, Hurst JR, Thomas SL, Smeeth L, Hubbard RB, Brown J, Quint JK. Bronchiectasis and the risk of cardiovascular disease: a population-based study. Thorax. 2017;72:161-6.

5. Evans IE, Bedi P, Quinn TM, Hill AT. Bronchiectasis severity is an independent risk factor for vascular disease in a bronchiectasis cohort. Chest. 2017:151:383-8.

6. Chen YF, Lin HH, Lin CS, Turbat B, Wang KA, Chung WS. Bronchiectasis and increased risk of ischemic stroke: a nationwide population-based cohort study. Int J Chron Obstruct Pulmon Dis. 2017;12:1375-83.

7. McDonnell MJ, Aliberti S, Goeminne PC, Restrepo MI, Finch S, Pesci A, Dupont LJ, Fardon TC, Wilson R, Loebinger MR, et al. Comorbidities and the risk of mortality in patients with bronchiectasis: an international multicentre cohort study. Lancet Respir Med. 2016:4:969-79.

8. Fuschillo S, Martucci M, Donner CF, Balzano G. Airway bacterial colonization: the missing link between COPD and cardiovascular events? Respir Med. 2012;106:915-23.

9. Vlachopoulos C, Xaplanteris P, Aboyans V, Brodmann M, Cifkova R, Cosentino F, De Carlo M, Gallino A, Landmesser U, Laurent S, et al. The role of vascular biomarkers for primary and secondary prevention. A position paper from the European Society of Cardiology Working Group on peripheral circulation: endorsed by the Association for Research into arterial structure and physiology (ARTERY) society. Atherosclerosis. 2015;241:507-32.

10. Cavalcante JL, Lima JA, Redheuil A, Al-Mallah MH. Aortic stiffness: current understanding and future directions. J Am Coll Cardiol. 2011:57:1511-22.

11. Laurent S, Boutouyrie P, Asmar R, Gautier I, Laloux B, Guize L, Ducimetiere P, Benetos A. Aortic stiffness is an independent predictor of all-cause and cardiovascular mortality in hypertensive patients. Hypertension. 2001;37:1236-41.

12. Laurent S, Cockcroft J, Van Bortel L, Boutouyrie P, Giannattasio C, Hayoz D, Pannier B, Vlachopoulos C, Wilkinson I, Struijker-Boudier H. Expert consensus document on arterial stiffness: methodological issues and clinical applications. Eur Heart J. 2006:27:2588-605.

13. Tanaka H, Munakata M, Kawano Y, Ohishi M, Shoji T, Sugawara J, Tomiyama H, Yamashina A, Yasuda H, Sawayama T, et al. Comparison between carotid-femoral and brachial-ankle pulse wave velocity as measures of arterial stiffness. J Hypertens. 2009;27:2022-7.

14. Chen R, He W, Zhang K, Zheng H, Lin L, Nie R, Wang J, Huang H. Airflow obstruction was associated with elevation of brachial-ankle pulse wave velocity but not ankle-brachial index in aged patients with chronic obstructive pulmonary disease. Atherosclerosis. 2015;242:135-40.

15. Wang LY, Zhu YN, Cui JJ, Yin KQ, Liu SX, Gao YH. Subclinical atherosclerosis risk markers in patients with chronic obstructive pulmonary disease: a systematic review and meta-analysis. Respir Med. 2017;123:18-27.

16. Sun WX, Jin D, Li Y, Wang RT. Increased arterial stiffness in stable and severe asthma. Respir Med. 2014;108:57-62.

17. Chalmers JD, Smith MP, McHugh BJ, Doherty C, Govan JR, Hill AT. Short- and long-term antibiotic treatment reduces airway and systemic inflammation in non-cystic fibrosis bronchiectasis. Am J Respir Crit Care Med. 2012:186:657-65

18. Gale NS, Bolton CE, Duckers JM, Enright S, Cockcroft JR, Shale DJ. Systemic comorbidities in bronchiectasis. Chron Respir Dis. 2012;9:231-8.

19. Martinez-Garcia MA, de Gracia J, Vendrell Relat M, Giron RM, Maiz Carro L, de la Rosa CD, Olveira C. Multidimensional approach to non-cystic fibrosis bronchiectasis: the FACED score. Eur Respir J. 2014;43:1357-67.

20. Chalmers JD, Goeminne P, Aliberti S, McDonnell MJ, Lonni S, Davidson J, Poppelwell L, Salih W, Pesci A, Dupont LJ, et al. The bronchiectasis severity index. An international derivation and validation study. Am J Respir Crit Care Med. 2014;189:576-85.

21. Venning V, Bartlett J, Jayaram L. Patients hospitalized with an infective exacerbation of bronchiectasis unrelated to cystic fibrosis: clinical, physiological and sputum characteristics. Respirology. 2017;22:922-7.

22. Guan WJ, Gao YH, Xu G, Lin ZY, Tang Y, Li HM, Lin ZM, Jiang M, Zheng JP, Chen $\mathrm{RC}$, et al. Inflammatory responses, spirometry, and quality of life in subjects with bronchiectasis exacerbations. Respir Care. 2015;60:1180-9.

23. Gao YH, Guan WJ, Xu G, Lin ZY, Tang Y, Lin ZM, Gao Y, Li HM, Zhong NS, Zhang GJ, et al. The role of viral infection in pulmonary exacerbations of bronchiectasis in adults: a prospective study. Chest. 2015;147:1635-43.

24. Guan WJ, Gao YH, Xu G, Lin ZY, Tang Y, Li HM, Lin ZM, Zheng JP, Chen RC, Zhong NS. Aetiology of bronchiectasis in Guangzhou, southern China. Respirology. 2015:20:739-48.

25. Gao YH, Guan WJ, Liu SX, Wang L, Cui JJ, Chen RC, Zhang GJ. Aetiology of bronchiectasis in adults: a systematic literature review. Respirology. 2016;21:1376-83.

26. Miller MR, Hankinson J, Brusasco V, Burgos F, Casaburi R, Coates A, Crapo R, Enright P, van der Grinten CP, Gustafsson P, et al. ATS/ERS task force: standardization of spirometry. Eur Respir J. 2005:26:319-38.

27. Vlachopoulos C, Aznaouridis K, Terentes-Printzios D, loakeimidis N, Stefanadis C. Prediction of cardiovascular events and all-cause mortality with brachial-ankle elasticity index: a systematic review and meta-analysis. Hypertension. 2012;60:556-62.

28. Aksamit TR, O'Donnell AE, Barker A, Olivier KN, Winthrop KL, Daniels MLA, Johnson M, Eden E, Griffith D, Knowles M, Metersky M, et al. Adult patients with bronhchiectasis: a first look at the US bronchiectasis research registry. Chest. 2017;151:982-92.

29. Aliberti S, Hill AT, Mantero M, Battaglia S, Centanni S, Lo Cicero S, Lacedonia D, Saetta M, Chalmers JD, Blasi F. Quality standards for the management of bronchiectasis in Italy: a national audit. Eur Respir J. 2016:48:244-8.

30. Buehler T, Steinmann M, Singer F, Regamey N, Casaulta C, Schoeni MH, Simonetti GD. Increased arterial stiffness in children with cystic fibrosis. Eur Respir J. 2012;39:1536-7. 
31. Aliberti S, Lonni S, Dore S, McDonnell MJ, Goeminne PC, Dimakou K, Fardon TC, Rutherford R, Pesci A, Restrepo MI, et al. Clinical phenotypes in adult patients with bronchiectasis. Eur Respir J. 2016;47:1113-22.

32. Finch $\mathrm{S}, \mathrm{McD}$ onnell MJ, Abo-Leyah $\mathrm{H}$, Aliberti S, Chalmers JD. A comprehensive analysis of the impact of Pseudomonas aeruginosa colonization on prognosis in adult bronchiectasis. Ann Am Thorac Soc. 2015;12:1602-11.

33. Guan WJ, Jiang M, Gao YH, Li HM, Xu G, Zheng JP, Chen RC, Zhong NS. Unsupervised learning technique identifies bronchiectasis phenotype with distinct clinical characteristics. Int I Tuberc Lung Dis. 2016;20:402-10.

34. Patel AR, Kowlessar BS, Donaldson GC, Mackay AJ, Singh R, George SN, Garcha DS, Wedzicha JA, Hurst JR. Cardiovascular risk, myocardial injury, and exacerbations of chronic obstructive pulmonary disease. Am J Respir Crit Care Med. 2013;188:1091-9.

35. Zieman SJ, Melenovsky V, Kass DA. Mechanisms, pathophysiology, and therapy of arterial stiffness. Arterioscler Thromb Vasc Biol. 2005;25:932-43.

36. Saleh AD, Chalmers JD, De Soyza A, Fardon TC, Koustas SO, Scott J, Simpson AJ, Brown JS, Hurst JR. The heterogeneity of systemic inflammation in bronchiectasis. Respir Med. 2017;127:33-9.

37. Vestbo J, Agusti A, Wouters EF, Bakke P, Calverley PM, Celli B, Coxson H, Crim C, Edwards LD, Locantore N, et al. Should we view chronic obstructive pulmonary disease differently after ECLIPSE? A clinical perspective from the study team. Am J Respir Crit Care Med. 2014;189:1022-30.

38. Zhao X, Wang H, Bo L, Zhao H, Li L, Zhou Y. Serum lipid level and lifestyles are associated with carotid femoral pulse wave velocity among adults: 4.4-year prospectively longitudinal follow-up of a clinical trial. Clin Exp Hypertens. 2017;16:1-8.

39. Loehr LR, Meyer ML, Poon AK, Selvin E, Palta P, Tanaka H, Pankow JS, Wright JD, Griswold ME, Wagenknecht LE, et al. Prediabetes and diabetes are associated with arterial stiffness in older adults: the ARIC study. Am J Hypertens. 2016;29:1038-45.

40. Tomiyama H, Hashimoto H, Tanaka H, Matsumoto C, Odaira M, Yamada J, Yoshida M, Shiina K, Nagata M, Yamashina A. Continuous smoking and progression of arterial stiffening: a prospective study. J Am Coll Cardiol. 2010;55:1979-87.

41. Tabara Y, Muro S, Takahashi Y, Setoh K, Kawaguchi T, Terao C, Kosugi S, Sekine A, Yamada R, Nakayama T, et al. Airflow limitation in smokers is associated with arterial stiffness: the Nagahama study. Atherosclerosis. 2014;232:59-64

42. Cecelia M, Chowienczyk P. Dissociation of arotic pulse wave velocity with risk factors for cardiovacular disease other than hypertension: a systematic review. Hypertension. 2009;54:1328-36.

43. Berry CL, Greenwald SE. Effects of hypertension on the static mechanical properties and chemical composition of the rat aorta. Cardiovasc Res. 1976;10:437-51.

44. Chalmers JD. Bronchiectasis and COPD overlap: a case of mistaken identity? Chest. 2017;151:1204-6.

45. Cauwenberghs N, Knez J, Tikhonoff V, D'hooge J, Kloch-Badelek M, Thijs L, Stolarz-Skrzypek K, Haddad F, Wojciechowska W, Swierblewska E, et al. Doppler indexes of left ventricular systolic and diastolic function in relation to the arterial stiffness in a general population. J Hypertens. 2016;34:762-71.

\section{Ready to submit your research? Choose BMC and benefit from:}

- fast, convenient online submission

- thorough peer review by experienced researchers in your field

- rapid publication on acceptance

- support for research data, including large and complex data types

- gold Open Access which fosters wider collaboration and increased citations

- maximum visibility for your research: over $100 \mathrm{M}$ website views per year

At BMC, research is always in progress.

Learn more biomedcentral.com/submissions 